\title{
High-Field Electronic Properties of Graphene
}

\author{
U. Zeitler • A.J.M. Giesbers • A. McCollam • \\ E.V. Kurganova · H.J. van Elferen · J.C. Maan
}

Received: 22 July 2009 / Accepted: 1 December 2009 / Published online: 6 January 2010

(C) The Author(s) 2010. This article is published with open access at Springerlink.com

\begin{abstract}
We have measured the energy gaps in single-layer and bilayer graphene by means of temperature dependent transport experiments in high magnetic fields up to $33 \mathrm{~T}$. They follow the expected Landau level splitting when a finite level width is taken into account. The quantum Hall effect, hitherto only observed up to $30 \mathrm{~K}$, remains visible up to $200 \mathrm{~K}$ in bilayers and even up to room temperature in singlelayer graphene. Our experiments in single-layer graphene show that the lowest Landau level, shared equally between electrons and holes at zero energy, becomes extremely narrow in high magnetic fields. It is this narrowing, together with the large Landau level splitting in graphene that leads to an extremely robust localization and makes the quantum Hall effect visible up to room temperature. In high magnetic fields $(B>20 \mathrm{~T})$ we observe a strongly increasing resistance with decreasing temperature. These results are explained with field dependent splitting of the lowest Landau level of the order of a few Kelvin, as extracted from activated transport measurements.
\end{abstract}

Keywords Graphene · Quantum Hall effect · Landau levels

\section{Introduction}

New material systems can sometimes yield new discoveries. A prominent example is graphene [1], the purely two-dimensional form of carbon arranged in a honeycomb lattice. Though graphene was known for a long time as the basic assembly part of graphite and carbon nanotubes it was long believed to be thermodynamically instable in its isolated form [2]. Only very recently purely two-dimensional single-layer graphene (SLG) was produced using mechanical exfoliation of graphite [3, 4], a discovery which opened an entirely new research field $[1,5]$.

U. Zeitler (凶) · A.J.M. Giesbers · A. McCollam · E.V. Kurganova · H.J. van Elferen · J.C. Maan High Field Magnet Laboratory, Institute for Molecules and Materials, Radboud University Nijmegen, Toernooiveld 7, 6525 ED Nijmegen, The Netherlands

e-mail: u.zeitler@science.ru.nl 
Fig. 1 (Color online) Dispersion of SLG (a) and BLG (b) around the Dirac point. The shaded regions sketch the occupied states when the Fermi energy is at the CNP. The bottom panels show the Landau level spectrum for SLG (c) and BLG (d)
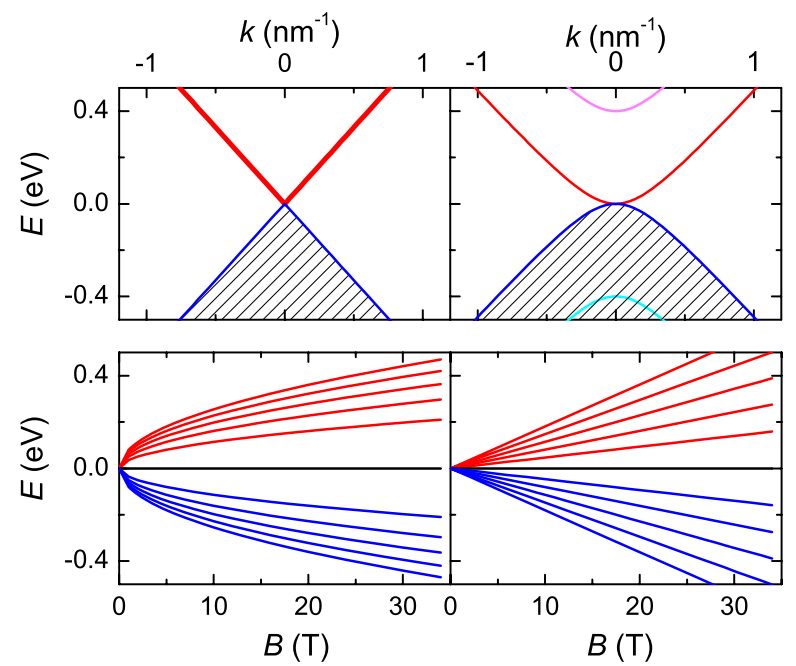

SLG is a zero-gap semiconductor where the conductance band and the valance band touch at the $K$-points of the Brillouin zone [6]. The charge carriers exhibit a linear dispersion, $E= \pm c p$, where $\mathbf{p}=\hbar\left(\mathbf{k}-\mathbf{k}_{\mathbf{0}}\right)$ is the quasi-momentum with respect to the $K$-point at $\mathbf{k}=\mathbf{k}_{\mathbf{0}}$ and the sign accounts for the charge of the particles (electrons or holes), see Fig. 1a. An additional topological degree of freedom, the socalled chirality, results from the fact that the honeycomb lattice of graphene consist of two triangular sublattices; the total wavefunction is a superposition of states from these two sublattices. Therefore, the linear dispersion observed in SLG mimics the behaviour of chiral massless Dirac fermions where the energy-independent particle velocity, $c=\sqrt{3} \gamma_{0} a / 2 \hbar \approx 10^{6} \mathrm{~m} / \mathrm{s}$ is determined by the exchange coupling $\gamma_{0}=$ $3.2 \mathrm{eV}$ and the lattice constant $a=0.246 \mathrm{~nm}$.

When stacking two layers of graphene on top of each other to form bilayer graphene (BLG), their inter-layer coupling $\gamma_{1} \approx 0.4 \mathrm{eV}$ induces a distortion of the linear dispersion around the $K$-points, resulting in a hyperbolic dispersion (Fig. 1c) [7]: $E(p)= \pm 1 / 2 \gamma_{1} \pm\left(1 / 4 \gamma_{1}^{2}+c^{2} \hbar^{2} p^{2}\right)^{1 / 2}$, which mimics the behaviour of massive, chiral, ultra-relativistic particles with a effective rest mass $m^{*}=\gamma_{1} / 2 c^{2} \approx 0.035 m_{e}$. For low energies, $E \ll \gamma_{1}$, the particles behave as in a conventional semiconductor with a parabolic dispersion $E(p)=p^{2} / 2 m^{*}$.

The relativistic properties of graphene are revealed most clearly in high magnetic fields. Here the energy spectrum splits up into non-equidistant Landau levels (Fig. 1c and 1d) with energies given by

$$
E_{N}^{S L G}=\operatorname{sgn}(N) \sqrt{2 \hbar c^{2} e B|N|} \quad \text { and } \quad E_{N}^{B L G}=\operatorname{sgn}(N) \frac{\hbar e B}{m^{*}} \sqrt{N(N-1)}
$$

in SLG [8] and BLG [7, 9], respectively. In both cases, $N$ is a negative integer for holes and positive for electrons. Cyclotron resonance experiments have indeed confirmed this Landau level structure in SLG [10] and BLG [11].

In SLG the $N=0$ Landau level is situated at zero energy (independent of the magnetic field). Like all other Landau levels with $|N| \geq 1$ it is fourfold degenerate but it 
is shared equally between electrons and holes of different chirality. In BLG the zeroenergy Landau level is eightfold degenerate and shared between charge carriers with $N=0$ and $|N|=1$, and all the other Landau levels $(|N| \geq 2)$ are fourfold degenerate.

Due to these relativistic Landau-level structures the well-known integer quantum Hall effect (QHE) [12, 13] observed in conventional two-dimensional electron systems with a parabolic dispersion transforms to a half-integer QHE in SLG [14, 15] and a to new type of unconventional integer quantum Hall effect in BLG [9].

This paper presents recent QHE experiments performed in magnetic fields up to $33 \mathrm{~T}$ at the High Field Magnet Laboratory in Nijmegen. We first review briefly the observation of the new types of QHEs in graphene. More specifically, we use activated transport experiments to map out the unconventional Landau-level spectrum of SLG and BLG. We show in particular that the gap between the zeroth and the first Landau level in SLG approaches the bare Landau-level separation for high magnetic fields, which is explained by a narrowing of the zero-energy Landau level [16]. For high magnetic fields and low temperatures, this narrow zeroth Landau level splits up into two levels separated by an activation gap of about $30 \mathrm{~K}$ at $30 \mathrm{~T}$ [17]. In contrast, the measured activation gaps between higher Landau levels are consistent with a large level broadening in both SLG and BLG and no splitting of these levels is observed.

\section{Quantum Hall Effects in Graphene}

Our samples are monolayer and bilayer graphene devices fabricated by micromechanical exfoliation of graphite [1,3], deposited on $\mathrm{Si} / \mathrm{SiO}_{2}$ substrate and patterned into a Hall bar (inset of Fig. 2a). Prior to patterning, the layer thickness was identified using optical microscopy [18] and atomic force microscopy. The carrier concentration $n$ in the graphene can be adjusted to either holes $(n<0)$ or electrons $(n>0)$ by applying a voltage $V_{g}$ on the highly doped Si-backgate: $n=C V_{g} / A$, were $C$ is the gate-capacitance and $A$ is the area of the graphene device. All measurements were preformed with standard AC-techniques at sufficiently low currents to avoid heating effects.

Figure 2 shows the QHE in SLG (left) and BLG (right) at a constant magnetic field $B=15 \mathrm{~T}$. When sweeping the carrier concentration, quantized plateaus appear in the Hall conductivity $\sigma_{x y}=i\left(4 e^{2} / h\right)$ at filling factors $v=4 i$, the factor 4 accounts for the degeneracy of the Landau level due to spin and chirality. The plateaus are accompanied by zero-minima in the longitudinal resistivity $\rho_{x x}$. In contrast to conventional electron systems, where $i$ is an integer $[12,13]$ the quantization occurs at half-integer values for SLG, i.e. at $v= \pm 2, \pm 6, \pm 10, \ldots$ In BLG, integer quantization is recovered with quantum-Hall plateaus at $v= \pm 4, \pm 8, \pm 12, \ldots$; however, the $i=0(v=0)$ plateau is missing. This unconventional integer QHE in BLG [9] can be attributed to the coexistence of electrons and holes in an eight fold-degenerate Landau level at zero energy.

To determine the energy gaps between adjacent Landau levels, we have measured the temperature dependence of the $\rho_{x x}$ minima. They follow an Arrhenius behaviour, $\rho_{x x} \propto \exp (-\Delta / 2 k T)$, where $\Delta$ is the distance between the levels reduced by the level width. In Fig. 3 we show the results for the measured gaps in SLG and BLG and 

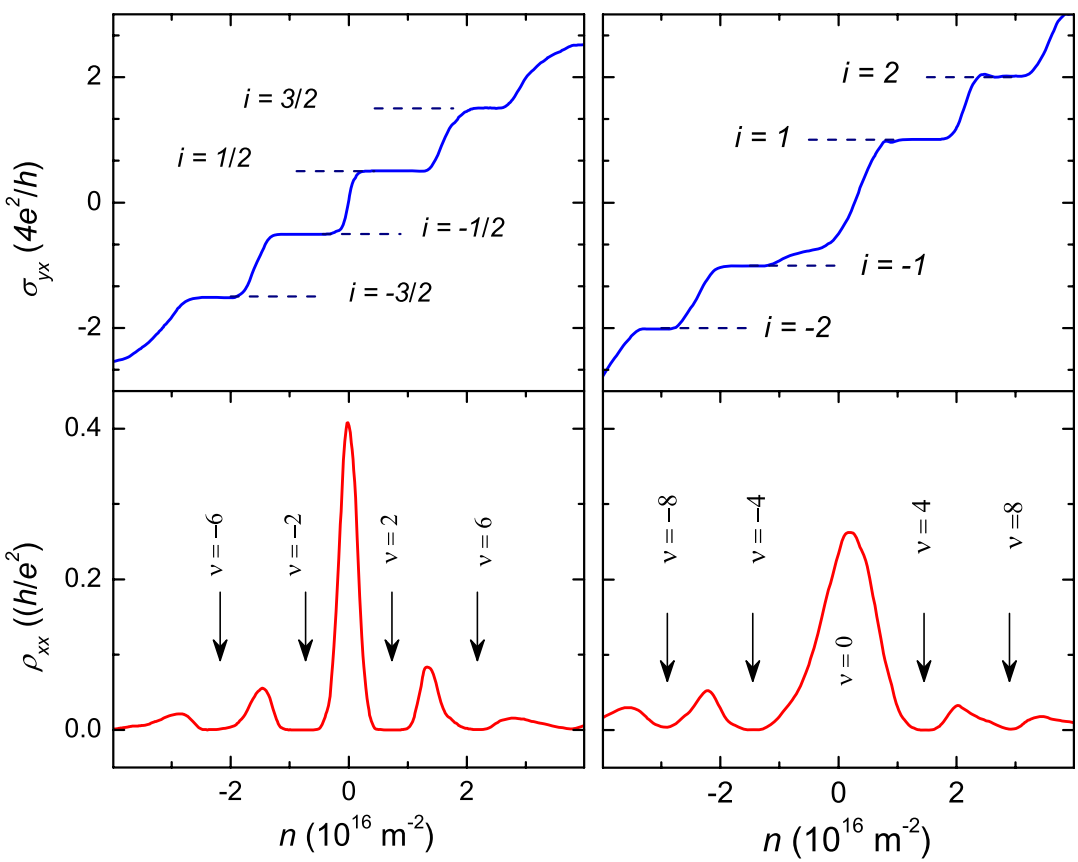

Fig. 2 (Color online) Hall conductivity $\sigma_{x y}$ (top) and magneto-resistivity $\rho_{x x}$ in SLG (left) and BLG (right) as a function of concentration at $B=15 \mathrm{~T}$ and $T=2 \mathrm{~K}$. The arrows mark the filling factors where quantum-Hall plateaus accompanied by Shubnikov-de Haas minima appear

Fig. 3 (Color online) Measured activation gaps for $v=2$ and $v=6$ in SLG (a) and for $v=4$ and $v=8$ in BLG (b). The (red) filled symbols represent values measured for electrons, (blue) open symbols are for holes. The lines are the bare level splittings, $E_{N+1}-E_{N}$, as predicted by (1) using $c=10^{6} \mathrm{~m} / \mathrm{s}$ for SLG and $m^{*}=0.07 m_{0}$ foe BLG

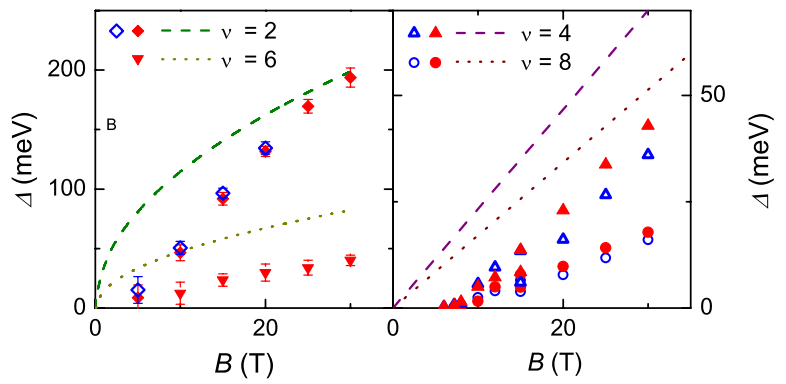

compare them to the bare Landau-level separation as given by (1). In SLG (Fig. 3a), the experimental values for $v=6$ show a constant offset of $\sim 400 \mathrm{~K}$ from the ideal curve, which can straightforwardly be explained by a corresponding finite Landaulevel width [19]. In contrast, the behaviour of the $v= \pm 2$ gap is strikingly different. At low magnetic fields, a lower value than the expected Landau level splitting is measured, but for high magnetic fields the measured gap approaches the bare Landaulevel separation. Since the results at $v=6$ show that the $N=1$ Landau level behaves as expected, the behaviour at $v= \pm 2$ can only be explained by the nature of the $N=0$ Landau level. Indeed, as we have shown in more detail elsewhere [16], the zerothLandau level is insensitive to important broadening mechanisms such as inter-valley 
Fig. 4 (Color online) Conductivity $\sigma_{x x}$ (bottom) and Hall conductivity $\sigma_{x y}$ (top) of a SLG sample around the CNP at $B=30 \mathrm{~T}$ and different temperatures. The inset shows the field dependence of the gap extracted from the temperature dependence of the conductivity minimum at $v=0$

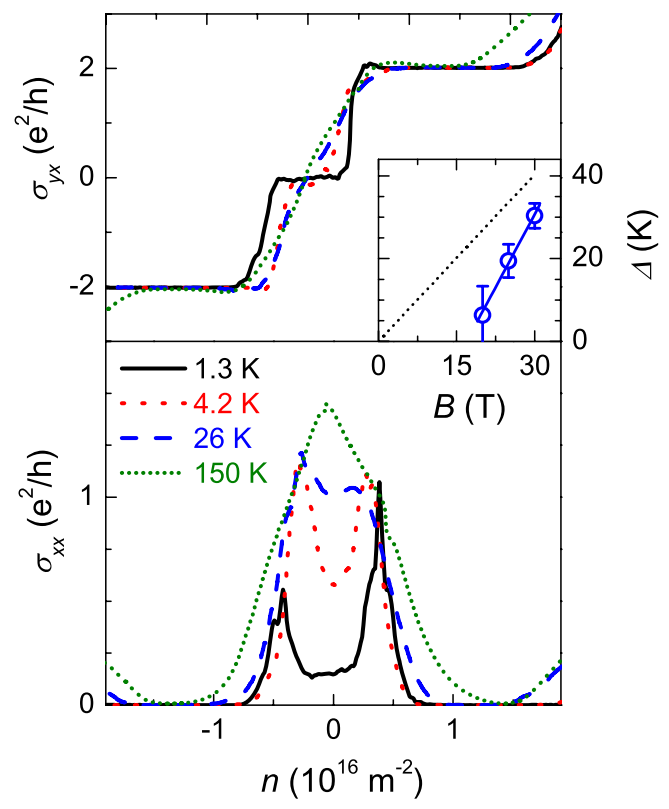

scattering and random magnetic-field fluctuations, and can become extremely narrow in high magnetic fields. Consequently, thermal excitations from $v=2$ minimum to this level reflect the bare level splitting. ${ }^{1}$

All activation gaps in BLG (Fig. 3b) depend linearly on the magnetic field (Fig. 1b and c) and they reasonably follow the Landau-level splitting $E_{N+1}-E_{N}$ as predicted by (1) with an effective mass about twice as high as naively expected. The additional offset can again be assigned to a finite level width. Interestingly, the slope for the two transitions deviates differently from the expectations which we tentatively explain by a linear field dependence of the Landau level widths [20]. Such an effect is expected for the higher Landau levels and can be related to random fluctuations of the local field orientation caused by the rippled surface of graphene [21]. Additionally, the deviations my also indicate a filling-factor dependent effective cyclotron mass as found in recent cyclotron resonance experiments [11].

The large activation gaps in graphene make it possible to observe a quantum Hall effect at high temperatures, up to $200 \mathrm{~K}$ in BLG and even up to room temperature in SLG [22]. This may pave the way towards using graphene as a high-temperature quantum resistance standard, first experiments indeed show promising results [23].

\section{High-Field Properties at the Charge Neutrality Point}

The peculiar behaviour of the zeroth Landau level around the charge neutrality point (CNP) in graphene is presently subject of intensive debate, see e.g. [5]. In this level,

\footnotetext{
${ }^{1}$ The thermally activated conductivity at $v=2$ is dominated by the $N=0$ Landau level with a considerably higher mobility than the $N=1$ level. Therefore, a broadening of the first Landau level is not seen in these experiments.
} 
electrons and holes of opposite chirality reside simultaneously in two possible spin states resulting in a four-fold degenerate level. In experiments performed on highmobility SLG (made from Kish-graphite) a lifting of this chiral degeneracy was observed by the appearance of a $v= \pm 1$ state [24, 25]. Most other experiments $[16,17,26-28]$ did not find such state, which points to a larger disorder preventing spontaneous symmetry breaking [29]. Instead, a divergence of $\rho_{x x}$ from its metallic value [26], $h / 4 e^{2}$, towards a large resistance $\rho_{x x} \gg h / e^{2}$ is observed.

Using the tensor inversions $\sigma_{x x}=\rho_{x x} /\left(\rho_{x x}^{2}+\rho_{x y}^{2}\right)$ and $\sigma_{x y}=-\rho_{x y} /\left(\rho_{x x}^{2}+\rho_{x y}^{2}\right)$, the divergence of $\rho_{x x}$ at low temperatures and high magnetic field together with a zero crossing of the Hall resistivity $\rho_{x y}$ engenders a zero minimum in $\sigma_{x x}$ and a plateau in $\sigma_{x y}$ quantized at zero value, see Fig. 4 . Note that this $v=0$ quantum-Hall state is distinctively different from the conventional QHE. Due to the zero crossing of $\rho_{x y}$ a diverging maximum in $\rho_{x x}$ leads to a zero minimum in $\sigma_{x x}$. Conventionally, one finds $\left|\rho_{x y}\right| \gg \rho_{x x}$ and quantum Hall states are characterized by a zero-minima of both the resistivity $\rho_{x x}$ and the conductivity $\sigma_{x x}$ and by non-zero quantized plateaus in $\rho_{x y}$ and $\sigma_{x y}$.

In order to uncover the origin of a diverging high-field resistance at the CNP we have measured the temperature dependence of the $\sigma_{x x}$ minimum at $v=0$ [17]. It reveals an activated behaviour, the corresponding gaps are plotted in the inset of Fig. 4. Such an emergence of a gap in the zeroth Landau level signifies a lifting of its fourfold degeneracy. Its linear field dependence suggests that the splitting might be spin related. Comparison with a Zeeman splitting using a free-electron $g$-factor $g=2$ [24], dashed line in the inset of Fig. 4, yields a reasonable agreement. The offset is due to a finite level width. The slightly higher slope of the experimentally measured gap may be explained by a sharpening of the zeroth Landau level, i.e. a reduction of its width with increasing field [16], or, alternatively by a slight exchange enhancement of the splitting.

Finally, it is interesting to remark that in BLG we have not yet observed clear signs of a low-temperature divergence in $\rho_{x x}$ and the opening of a gap in the lowest Landau level. Whether this is due to a large level broadening which would mask the formation of a gap still has to be explored experimentally.

\section{Conclusions}

In conclusion, we have measured the transport properties of SLG and BLG at low temperatures and high magnetic fields. We have shown that the quantum-Hall excitation gaps follow the expected behaviour when assuming a level broadening of a few hundred Kelvin. The zeroth Landau level in SLG, however, appeared to be extremely narrow, which made it possible to observe a field-induced splitting of this level.

The experiments presented in this work only form the tip of the iceberg and more remains to be done to fully uncover the fascinating electronic properties of graphene. And, undoubtedly, high magnetic field will continue to play an important role.

Acknowledgements All the experiments reported in this paper were performed on graphene samples fabricated at the University of Manchester. We would like to thank Kostya Novoselov, Andre Geim and 
the entire Manchester group for providing us with these excellent samples. We would also like to acknowledge theoretical input from Misha Katsnelson. This work is part of the research program of the 'Stichting voor Fundamenteel Onderzoek der Materie (FOM)', which is financially supported by the 'Nederlandse Organisatie voor Wetenschappelijk Onderzoek (NWO)'. It has also been supported by EuroMagNET under EU contract RII3-CT-2004-506239.

Open Access This article is distributed under the terms of the Creative Commons Attribution Noncommercial License which permits any noncommercial use, distribution, and reproduction in any medium, provided the original author(s) and source are credited.

\section{References}

1. A.K. Geim, K.S. Novoselov, Nat. Mater. 6, 183 (2007)

2. N.D. Mermin, Phys. Rev. 176, 250 (1968)

3. K.S. Novoselov et al., Science 306, 666 (2004)

4. K.S. Novoselov et al., Proc. Natl. Acad. Sci. USA 102, 10451 (2005)

5. A.H. Castro Neto et al., Rev. Mod. Phys. 81, 109 (2009)

6. P.R. Wallace, Phys. Rev. 71, 622 (1947)

7. E. McCann, V.I. Fal'ko, Phys. Rev. Lett. 96, 086805 (2006)

8. J.W. McClure, Phys. Rev. 104, 666 (1956)

9. K.S. Novoselov et al., Nat. Phys. 2, 177 (2006)

10. Z. Jiang et al., Phys. Rev. Lett. 98, 197403 (2007)

11. E.A. Henriksen et al., Phys. Rev. Lett. 100, 087403 (2008)

12. K. von Klitzing, Rev. Mod. Phys. 58, 519 (1985)

13. K. von Klitzing, G. Dorda, M. Pepper, Phys. Rev. Lett. 45, 494 (1980)

14. K.S. Novoselov et al., Nature 438, 197 (2005)

15. Y. Zhang, Y. Tan, H.L. Stormer, P. Kim, Nature 438, 201 (2005)

16. A.J.M. Giesbers et al., Phys. Rev. Lett. 99, 206803 (2007)

17. A.J.M. Giesbers et al., Phys. Rev. B 80, 201403 (2009)

18. P. Blake et al., Appl. Phys. Lett. 91, 063124 (2007)

19. T. Ando, A.B. Fowler, F. Stern, Rev. Mod. Phys. 54, 437 (1982)

20. E.V. Kurganova et al., to be published

21. J.C. Meyer et al., Nature 446, 60 (2007)

22. K.S. Novoselov et al., Science 315, 1379 (2007)

23. A.J.M. Giesbers et al., Appl. Phys. Lett. 93, 222109 (2008)

24. Y. Zhang et al., Phys. Rev. Lett. 96, 136806 (2006)

25. Z. Jiang, Y. Zhang, H.L. Stormer, P. Kim, Phys. Rev. Lett. 99, 106802 (2007)

26. D.A. Abanin et al., Phys. Rev. Lett. 98, 196806 (2007)

27. J.G. Checkelsky, L. Li, N.P. Ong, Phys. Rev. Lett. 100, 206801 (2008)

28. J.G. Checkelsky, L. Li, N.P. Ong, Phys. Rev. B 79, 115434 (2009)

29. K. Nomura, A.H. MacDonald, Phys. Rev. Lett. 96, 256602 (2006) 